\title{
Regional differences in the prevalence of oral allergy syndrome among Japanese children: A questionnaire-based survey
}

\author{
Masaya Ota,${ }^{1,2}$ Yutaka Nishida, ${ }^{1}$ Hisako Yagi, ${ }^{1}$ Koichiro Sato, ${ }^{1}$ Satoshi Yamada, ${ }^{1}$ Hirokazu Arakawa,,${ }^{1}$ Takumi Takizawa ${ }^{1}$
}

\begin{abstract}
Background: Oral allergy syndrome (OAS) is characterized by an immediate allergic reaction that mainly or partially affects the oral mucosa, pharynx, or lips, and it is usually caused by ingesting fresh fruits or vegetables. Most patients with OAS also have allergic rhinitis due to pollen. As allergic rhinitis is increasingly prevalent in the Japanese population and the age at disease development is decreasing, morbidity associated with OAS among the younger population is likely to increase. However, there is little information about the prevalence of this disease among Japanese children, specifically the influences of residency in regions with different environments.
\end{abstract}

Objectives: To investigate the prevalence of OAS and seasonal allergic rhinitis (SAR) among Japanese children and evaluate the relationship between OAS and SAR.

Methods: We administered a questionnaire-based survey among children aged 7-15 years, living in 4 cities in central Japan.

Results: The questionnaires were administered to 4103 children and completed by 3365 (82.0\%). Overall, 524 children (15.6\%) reported OAS-like symptoms after ingesting fruits or vegetables. The prevalence of seasonal SAR and oral symptoms significantly differed among the 4 cities. The total prevalence of oral symptoms co-occurring with SAR was $24.4 \%$, which was significantly higher than the prevalence of symptoms occurring without SAR $(10.2 \%, p<0.001)$.

Conclusion: Herein, oral symptoms were more likely to occur in patients with SAR than in those without SAR. The prevalence of SAR and food-induced oral symptoms significantly differed among the regions, suggesting they might be affected by regional differences in lifestyles and flora.

Key words: oral allergy syndrome, child, allergic rhinitis, prevalence, pollen-food allergy syndrome

\footnotetext{
From:

Gunma University Graduate School of Medicine, Pediatrics, Maebashi, Japan

Niigata University Graduate School of Medical and Dental Sciences, Department of Pediatrics, Niigata, Japan
}

\section{Introduction}

Oral allergy syndrome (OAS) is an immunoglobulin (Ig) E-mediated immediate type of food allergy whose symptoms are mainly localized to the mucous membrane within the oral cavity. ${ }^{1,2}$ Common causative foods associated with OAS are fresh fruits and vegetables. ${ }^{3}$ Moreover, OAS is often complicated by pollinosis; OAS that develops after sensitization to pollen is called pollen-associated food allergy syndrome or pollen-food allergy syndrome. ${ }^{4,5}$ Numerous studies have demonstrated a causative relationship between some pollen and OAS, for example, pollen of white birch and apples. Bet $\mathrm{v}$ 1, a pathogenesis-related protein (PR)-10 in birch pollen, has cross-allergenicity to Mal $\mathrm{d} 1$, which is a PR-10 in apples. ${ }^{6,7}$

\section{Corresponding author:}

Takumi Takizawa

3-39-22 Showa-machi, Maebashi, Japan

E-mail: takizawt@gunma-u.ac.jp

Indeed, a study based in Austria has reported that approximately $75 \%$ of patients with birch pollinosis present OAS symptoms after the ingestion of apples. ${ }^{8}$ Another prominent example involves mugwort pollen and celery. ${ }^{9}$ Cross-reactivity between tomato fruit and Japanese cedar pollen has also been reported. ${ }^{10}$

There are regional differences in the prevalence of seasonal allergic rhinitis (SAR) among adults. ${ }^{11}$ For instance, a questionnaire-based study of SAR prevalence in China showed a wide between-region variation in prevalence between adults ${ }^{12}$ and children. ${ }^{13}$ These differences are likely attributable to variations in environmental factors, such as climate and vegetation. ${ }^{14}$ 
As SAR affects the risk of OAS, the prevalence of OAS shows regional variation and is likely affected by environmental factors that influence SAR prevalence. For instance, OAS prevalence in Hyogo prefecture, Japan, is higher in regions abundant in Alnus sieboldiana trees than in other regions where this type of tree is rare. ${ }^{15}$ However, there have been few studies on the prevalence of OAS among Japanese children and its between-region variation. ${ }^{16,17}$ Therefore, the present study aimed to investigate the prevalence of OAS and SAR among Japanese children in regions with different climates and to examine the relationship between OAS and SAR. This is the first survey of SAR and OAS prevalence among children living in different prefectures in Japan.

\section{Methods}

Study design

We administered a structured questionnaire-based survey among school children aged 7-15 years in 4 cities in central Japan. The survey was administered between September 2015 and February 2016. Four cities in central Japan with different climatic characteristics were selected (Figure 1). Maebashi, in Gunma prefecture, is a city located at $108 \mathrm{~m}$ above sea level (asl), with approximately $1200 \mathrm{~mm}$ of annual rainfall and little snow in winter. Numata city, located approximately 30 $\mathrm{km}$ north of Maebashi, is set in a mountainous region at 493 $\mathrm{m}$ asl. Saku in Nagano prefecture is located in a mountainous region approximately $55 \mathrm{~km}$ west of Maebashi, at $683 \mathrm{~m}$ asl,
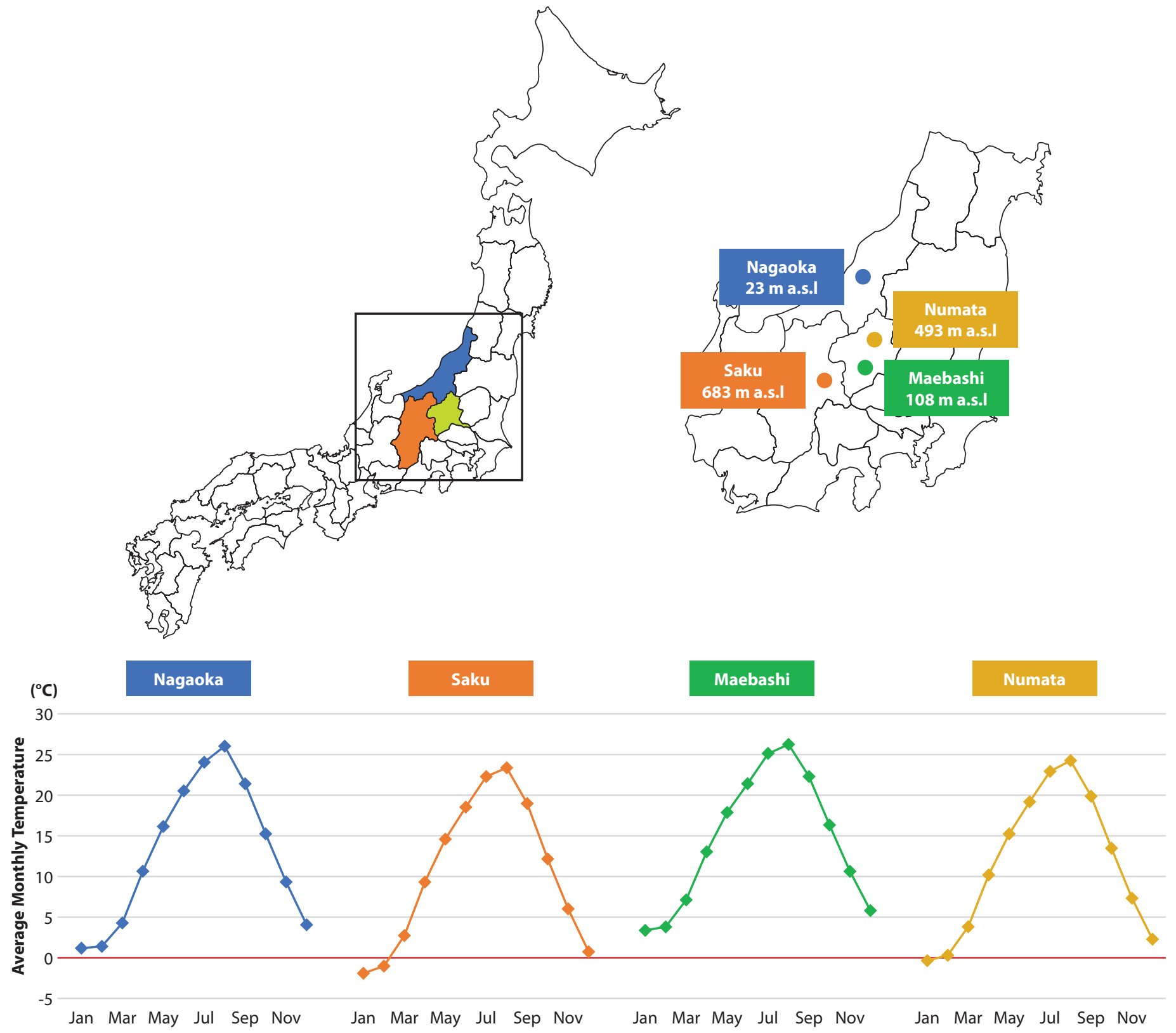

Figure 1. Profiles of the 4 cities in central Japan, including the altitude, average monthly temperature, and average monthly rainfall. Maebashi and Nagaoka are urban areas, whereas Saku and Numata are mountainous regions. asl, above sea level 


\begin{tabular}{l|l|l}
$(\mathrm{mm})$ & Saku & Maebashi \\
400 & &
\end{tabular}

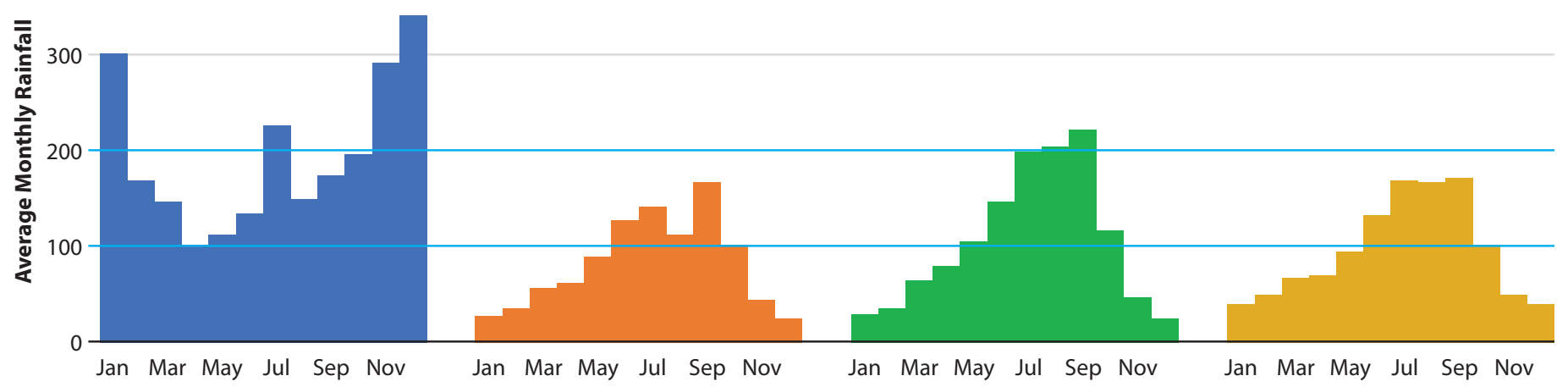

Figure 1. (Continued)

with winters colder than those in Numata. Nagaoka in Niigata prefecture is located approximately $120 \mathrm{~km}$ north of Maebashi and is a suburban city at $23 \mathrm{~m}$ asl, with the greatest annual snowfall among these four cities. Total forest areas in Maebashi, Numata, Nagaoka, and Saku are 7096, 33916, 34771, and 20165 hectares (ha), respectively. The coniferous forest areas in each city are 3,770 (53.1\% of total forest area), 15728 (46.4\%), $10862(31.2 \%)$, and 14216 ha (70.5\%), respectively. Forest of Japanese cedars and pine trees were only available in Saku and Nagaoka; Saku has 404 ha of Japanese cedars and 12803 ha of pine trees, while Nagaoka has 10663 ha and 171 ha, respectively. Among the 4 cities, only Saku has wild white birch trees. The burden seasons of the pollens of Japanese cedar, orchard grass, ragweed, and mugwort are similar in all the cities: February to April for Japanese cedar, May to August for orchard grass, and August to October for ragweed and mugwort. In each city, over 900 children from elementary schools and a junior high school participated in the study. Questionnaires were sent to 1 elementary and 1 junior high school in Maebashi, Numata, and Nagaoka, and to 3 elementary schools and 1 junior high school in Saku (Figure 1). Participation of 3 elementary schools was necessary to collect the questionnaires from $\sim 600$ students in Saku city, because the number of students in each elementary school in the city was small. Selection of schools was based on the recommendations of the $\mathrm{Mu}$ nicipal Board of Education in each city. The questionnaire was distributed among all students, who were asked to complete it with their guardians at home, and it was subsequently collected by their teachers. The study protocol was approved by the Ethics Committee of Gunma University (\#27-18). We gave prospective participants a document describing the study purpose, which informed them that participation was anonymous and that filling out a questionnaire would be considered to be indicative of consent to participation.

\section{Questionnaire}

We developed a questionnaire for this study that contained the following items:

1) What is your sex (boy or girl)?

2) What is your age?

3) Have you ever been diagnosed with seasonal allergic rhinitis (SAR) by an attending physician?
4) What kinds of pollen trigger your seasonal allergic rhinitis (multiple answers are permitted)?

5) Have you experienced any of the following symptoms after ingesting fruits or vegetables: itching; tingling; stinging; or numbness or swelling of the tongue, palate, mouth, ear, or throat? (If no, go to question 9.)

6) What type of fruit or vegetable causes any of the above symptoms (multiple answers are permitted)?

7) What kind of symptoms do you develop on the tongue, palate, lip, ear, or throat after ingestion of fruits or vegetables (multiple answers are permitted)?

8) What kind of symptoms, other than OAS-like symptoms, do you feel after ingesting fruits or vegetables?

9) Have you ever been diagnosed with any of the following allergic diseases by an attending physician: atopic dermatitis, bronchial asthma, perennial allergic rhinitis, or food allergy?

\section{Statistical analysis}

Chi-square tests were used to compare proportions between regions as categorical variables. The Cochran-Armitage test was used to evaluate age. Spearman correlation analysis was used to determine whether there were any correlations between the prevalence of SAR and oral symptoms. These statistical analyses were conducted using $\mathrm{R}$ version 3.2.2 revised ( $\mathrm{R}$ Core Development Team) ${ }^{18}$ Multiple logistic regression analysis was performed to calculate odds ratios (ORs) for the prevalence of oral symptoms between groups. These analyses were performed using SPSS statistics version 24 (IBM Corp.). The level of significance was set at $p<0.05$ for all tests.

\section{Results \\ Participants' characteristics}

Overall, there were 4103 children aged 7-15 years across the included schools. The questionnaires were returned by 3365 children $(82.0 \%)$ (Table 1). The number of responders was lower $(72.3 \%)$ in Saku than in other cities. There were no differences between the cities in participants' sex and age. The mean prevalence of allergic diseases, atopic dermatitis (AD), bronchial asthma, perennial allergic rhinitis, and food allergy (FA) was $16.3 \%, 18.5 \%, 28.8 \%$, and 6.0\%, respectively (Table $\mathbf{1}$ ). 
Table 1. Characteristics of children and allergic diseases in 4 Japanese cities

\begin{tabular}{|c|c|c|c|c|c|}
\hline & Maebashi & Numata & Saku & Nagaoka & Total \\
\hline Number of participants & 1100 & 1046 & 931 & 1026 & 4103 \\
\hline Responders (\%) & $929(84.5)$ & $870(83.2)$ & $673(72.3)$ & $893(87.0)$ & $3365(82.0)$ \\
\hline Sex, male (\%) & $458(49.4)$ & $404(46.6)$ & $341(50.7)$ & $436(49.0)$ & $1639(48.8)$ \\
\hline Mean age (years) & 11.0 & 11.0 & 11.1 & 10.9 & 11.0 \\
\hline \multicolumn{6}{|c|}{ Number of responders at each age, years old (\%) } \\
\hline 7 & $101(10.9)$ & $104(12.0)$ & $62(9.2)$ & $109(12.2)$ & $376(11.2)$ \\
\hline 8 & $102(11.0)$ & $72(8.3)$ & $67(10.0)$ & $99(11.1)$ & $340(10.1)$ \\
\hline 9 & $96(10.3)$ & $106(12.2)$ & $80(11.9)$ & $90(10.1)$ & $372(11.1)$ \\
\hline 10 & $97(10.4)$ & $89(10.2)$ & $73(10.8)$ & $119(13.3)$ & $378(11.2)$ \\
\hline 11 & $110(11.8)$ & $117(13.4)$ & $78(11.6)$ & $95(10.6)$ & $400(11.9)$ \\
\hline 12 & $106(11.4)$ & $104(12.0)$ & $86(12.8)$ & $92(10.3)$ & $388(11.5)$ \\
\hline 13 & $125(13.5)$ & $83(9.5)$ & $86(12.8)$ & $94(10.5)$ & $388(11.5)$ \\
\hline 14 & $118(12.7)$ & $85(9.8)$ & $46(6.8)$ & $119(13.3)$ & $368(10.9)$ \\
\hline 15 & $74(8.0)$ & $110(12.6)$ & $95(14.1)$ & $76(8.5)$ & $355(10.5)$ \\
\hline Atopic dermatitis (\%) & $165(17.8)$ & $116(13.3) *$ & $131(19.5)$ & $137(15.3)$ & $549(16.3)$ \\
\hline Bronchial asthma (\%) & $148(15.9)$ & $134(15.4)^{*}$ & $170(25.3)^{* * *}$ & $170(19.0)$ & $622(18.5)$ \\
\hline Perennial allergic rhinitis (\%) & $277(29.8)$ & $265(30.5)$ & $167(24.8)$ & $260(29.1)$ & $969(28.8)$ \\
\hline Food allergy (\%) & $66(7.1)$ & $53(6.1)$ & $46(6.8)$ & $36(4.0)^{*}$ & $201(6.0)$ \\
\hline Seasonal allergic rhinitis (\%) & $477(51.3)^{* * *}$ & $370(42.5)^{\star *}$ & $231(34.3)^{\star *}$ & $205(23.0)^{* * *}$ & $1283(38.1)$ \\
\hline Oral symptoms (\%) & $202(21.7)^{\star * *}$ & $119(13.7)$ & $97(14.4)$ & $106(11.9)^{*}$ & $524(15.6)$ \\
\hline
\end{tabular}

Chi-square test: ${ }^{*} p$-value $<0.05,{ }^{* *} p$-value $<0.01,{ }^{* * *} p$-value $<0.001$

A

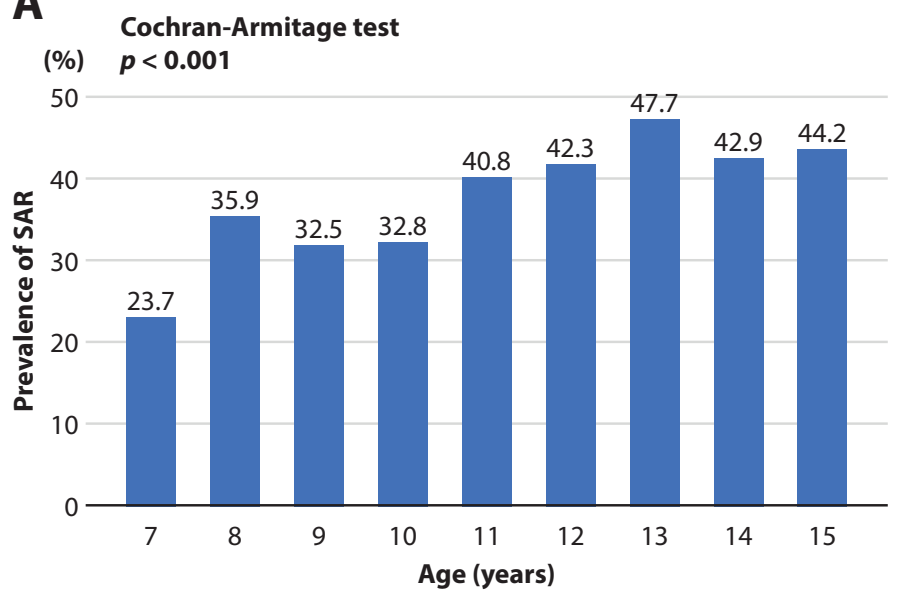

B
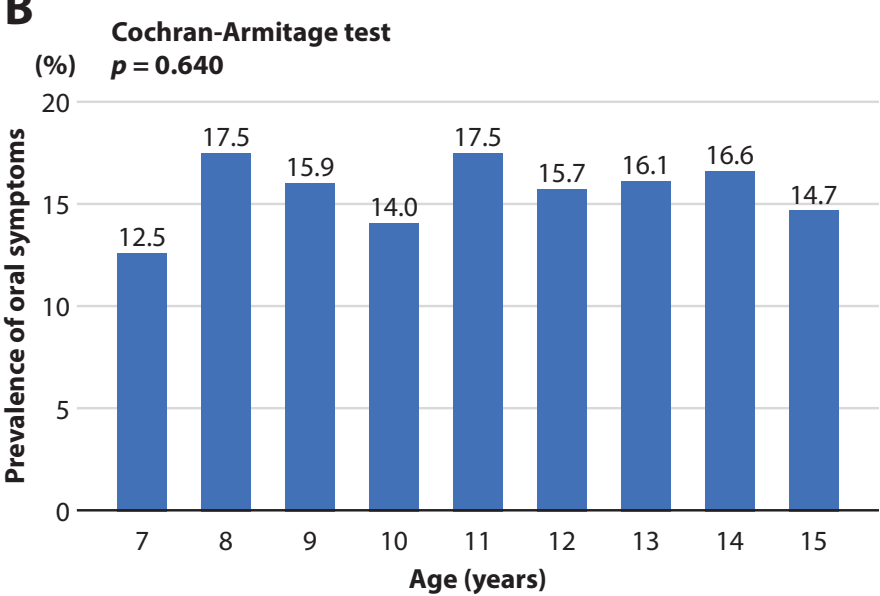

Figure 2. Prevalence of seasonal allergic rhinitis (SAR), oral symptoms, and other symptoms after ingesting fruits or vegetables Age-stratified prevalence of SAR, which increases with age (Cochran-Armitage test, $p<0.001)(\mathrm{A})$. Age-stratified prevalence of oral symptoms, which remains approximately constant across age groups (Cochran-Armitage test, $p=0.640)(B)$. Frequencies of oral symptoms among 524 participants (C). Frequencies of symptoms other than those of the oral cavity (D). 


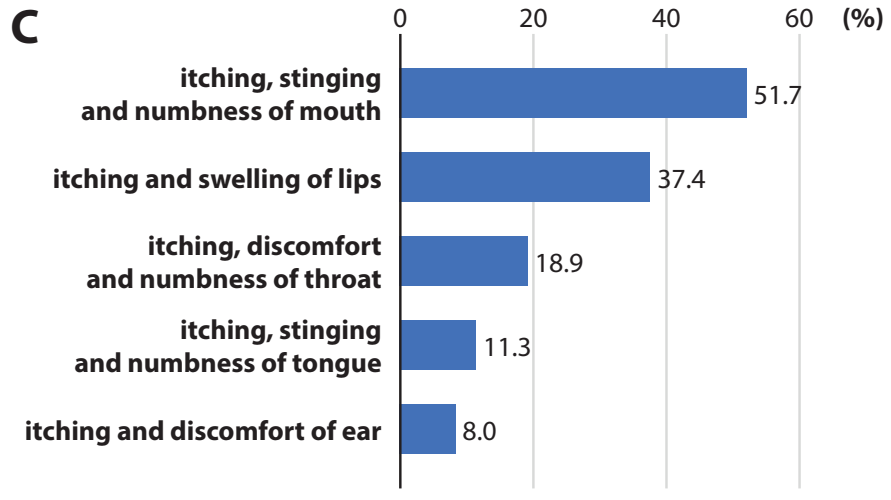

Figure 2. (Continued)

There were significant differences in the prevalence of $\mathrm{AD}$ in Numata city, asthma in Numata and Saku cities, and FA in Nagaoka (Table 1).

\section{Seasonal allergic rhinitis}

The average prevalence of SAR diagnosed by an attending physician was $38.1 \%(1283 / 3365)$. The prevalence of SAR increased with age (Figure 2A). The prevalence of SAR was the highest in Maebashi (51.3\%) (Table 1), and it significantly differed among the cities. Fourteen different types of pollen were reported as triggers of SAR (Table 2). The most frequently reported cause of SAR was Japanese cedar pollen. Among children with SAR, 75.3\% had been diagnosed as having SAR related to Japanese cedar (Table 2). Concurrently, white birch pollinosis, associated with Rosaceae fruit allergy, was diagnosed in only $1.0 \%$ of all children.

\section{Oral allergy syndrome-like symptoms}

The average prevalence of OAS-like symptoms (oral symptoms) after ingesting fruits or vegetables was 15.6\% (524/3365) (Table 1). This prevalence was the highest in Maebashi (21.7\%), and it differed significantly among the 4 cities (Table 1). There was no difference in the prevalence of oral symptoms between age groups (Figure $\mathbf{2 B}, p=0.640$ ). There were 39 different kinds of fruits and vegetables associated with oral symptoms. Fruits or vegetables frequently reported as being associated with OAS-like symptoms were kiwi fruit, pineapple, melon, Japanese yam, and watermelon. Of 524 children who reported oral symptoms, $341(65 \%)$ reported at least 1 fruit or vegetable as a cause, $95(18.1 \%)$ reported 2, and $44(8.4 \%)$ reported 3 different foods as symptom triggers. The largest number of food triggers reported by 1 child was 8 .

Children reported a range of oral symptoms after ingesting fruits or vegetables (Figure 2C). A combination of itching, tingling, stinging, and numbness of the mouth was the most frequently reported group of symptoms (51.7\%). It is worth noting that most children reported itching inside the mouth. Indeed, $87.0 \%$ of the children who experienced oral symptoms reported experiencing exclusively oral symptoms (Figure 2D). Concurrently, other symptoms including skin manifestations, such as urticarial and erythematous rash, respiratory symptoms, and abdominal symptoms, were reported by $9.7 \%, 2.9 \%$, and $1.7 \%$ of the surveyed children, respectively.

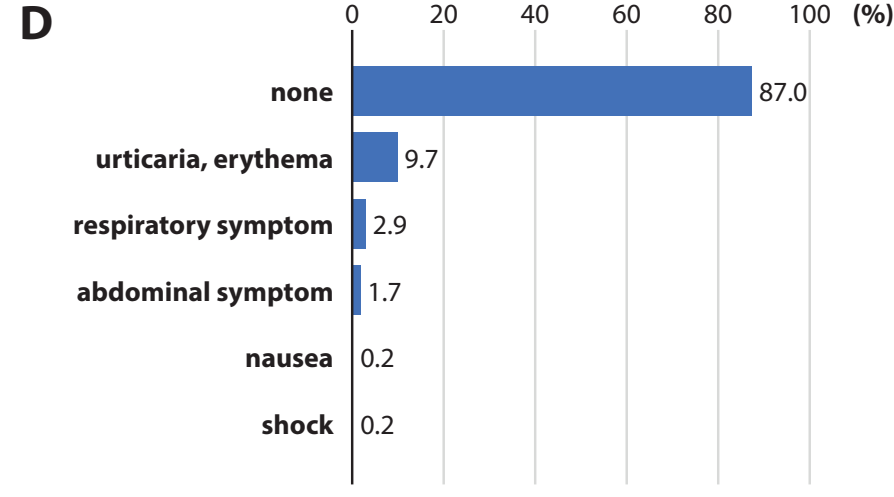

Table 2. Pollen types respondents with SAR were sensitized to

\begin{tabular}{|lcc|}
\hline \multicolumn{1}{c}{ Pollen } & $\begin{array}{c}\text { Number } \\
\text { (Total respondents with SAR 1283) }\end{array}$ & $\%$ \\
\hline Japanese cedar & 966 & 75.3 \\
\hline Ragweed & 279 & 21.7 \\
\hline Rice & 187 & 14.6 \\
\hline Cypress & 184 & 14.3 \\
\hline Orchard grass & 90 & 7.0 \\
\hline Sweet vernal grass & 23 & 1.8 \\
\hline Wormwood & 20 & 1.6 \\
\hline White birch & 13 & 1.0 \\
\hline Weed & 13 & 1.0 \\
\hline Timothy grass & 12 & 0.9 \\
\hline Japanese alder & 7 & 0.5 \\
\hline Pine tree & 4 & 0.3 \\
\hline Wheat & 4 & 0.3 \\
\hline Dandelion & 2 & 0.2 \\
\hline
\end{tabular}

Multiple answers were allowed.

Abbreviation: SAR, seasonal allergic rhinitis

\section{Association between SAR and OAS}

There was a correlation between the prevalence of SAR and oral symptoms in all regions $(\mathrm{R}=0.848)$ (Figure $3 \mathrm{~A}$ ). Moreover, oral symptoms were more common among SARdiagnosed than among non-SAR-diagnosed children (Figure 3B; $p<0.001)$. The sex- and city-adjusted OR for the prevalence of oral symptoms in SAR-diagnosed children was 2.751 (95\% confidence interval: 2.259-3.351) (Table 3). A similar association was observed in each city (Figure 3C). The prevalence of oral symptoms was higher among children with than among those without SAR across all cities. The highest prevalence of oral symptoms among the SAR-diagnosed children was found in Maebashi (Figure 3C). For most foods, the ratio of oral symptoms in those with SAR to that in all participants was more than 50\%, but Nagaimo yam and Mango showed lower ratios, indicating that the oral symptoms associated with those foods have a weaker association with allergic rhinitis. 

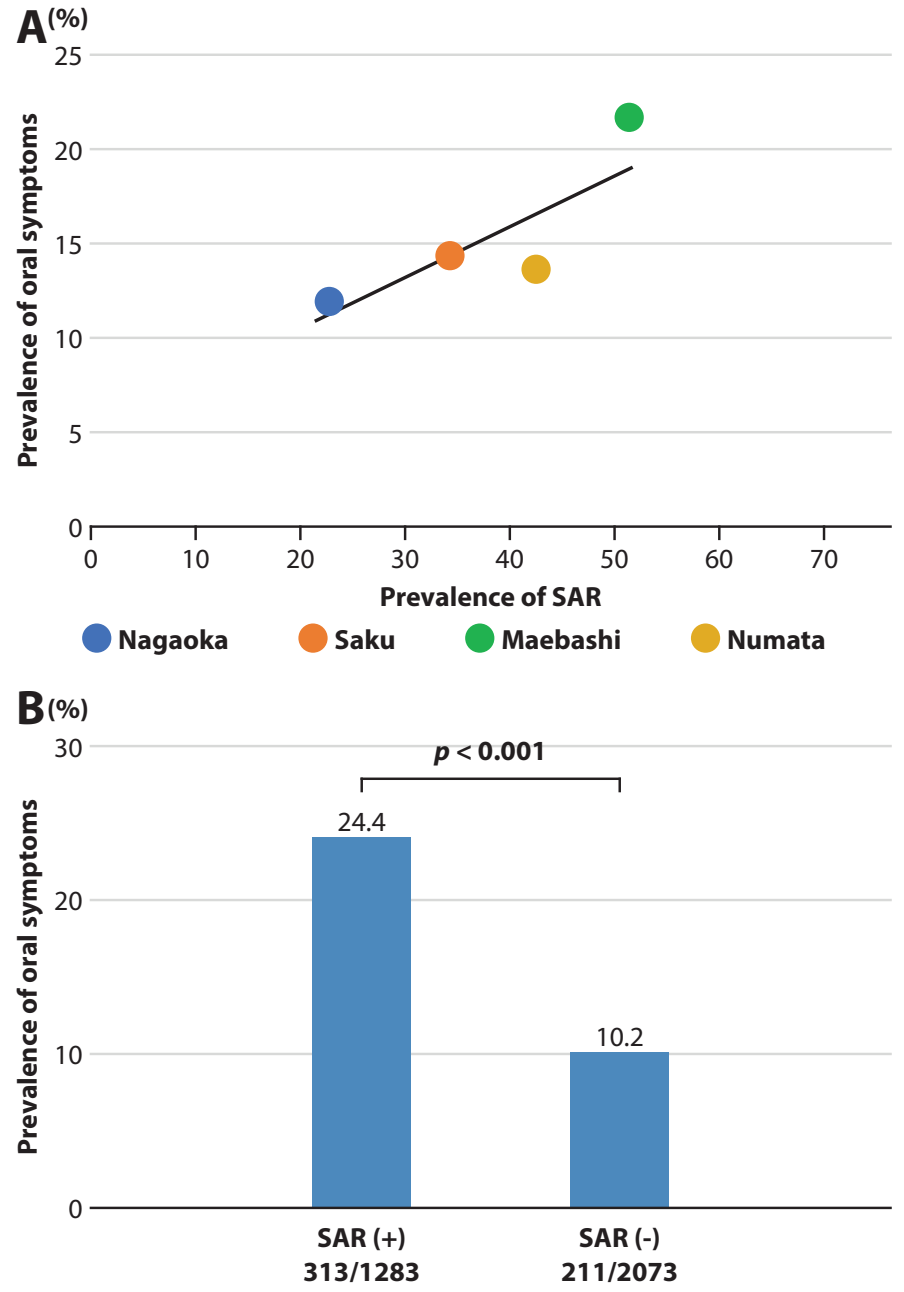

$\mathbf{C}_{(\%)}$

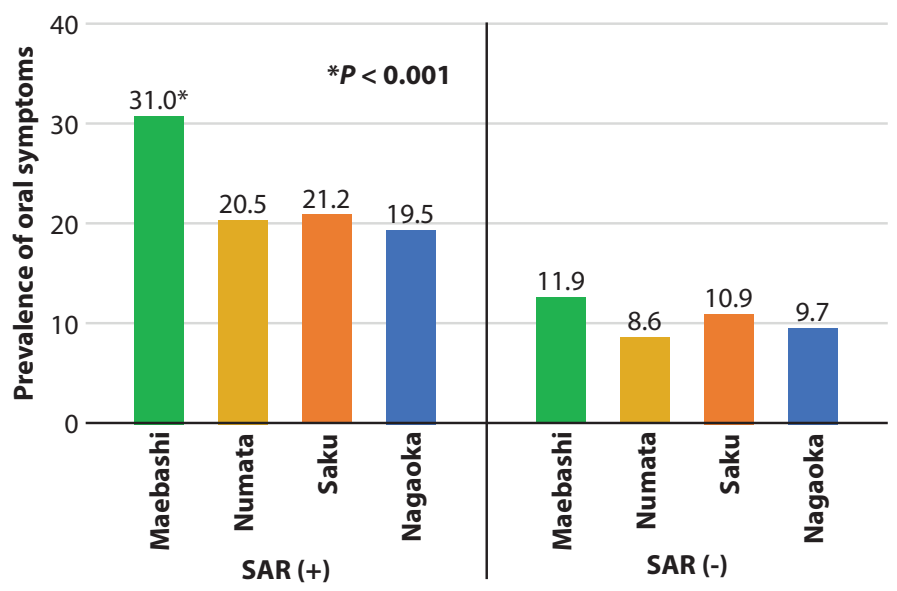

Figure 3. Correlation between seasonal allergic rhinitis (SAR) and oral symptoms

Correlation between the prevalence of SAR and oral symptoms across 4 cities. There is a linear association between the prevalence SAR and oral symptoms across 4 cities (Spearman's correlation analysis, $\left.y=0.3049 x+3.9053, R^{2}=0.7192\right)(A)$. Comparison of the overall prevalence of oral symptoms in children with and without SAR (chi-square test, $p<0.001$ ) (B). Prevalence of oral symptoms with or without SAR in different cities. Maebashi has a significantly higher prevalence of oral symptoms than that in other cities among children with SAR (chi-square test, $p<0.001)(\mathrm{C})$.
Table 3. Odds ratios and adjusted odds ratio for oral symptoms between children with and without seasonal allergic rhinitis

\begin{tabular}{lcccc} 
& \multicolumn{2}{c}{ Oral symptoms } & Odds & 95\% CI \\
\cline { 2 - 3 } & + & - & ratio & \\
SAR (+) & $313(24.4)$ & $968(75.5)$ & 2.913 & $2.404-3.530$ \\
& & & $*[2.751]$ & $*[2.259-3.351]$ \\
SAR (-) & $211(10.2)$ & $1858(89.8)$ & 1 & \\
\hline Sex & & & & \\
male & $224(13.7)$ & $1410(86.3)$ & 0.699 & $0.576-0.847$ \\
female & $300(17.5)$ & $1415(82.5)$ & 1 & \\
\hline City & & & & \\
\hline Maebashi & $202(21.7)$ & $727(78.3)$ & 1.402 & $1.067-1.840$ \\
\hline Numata & $119(13.7)$ & $751(86.3)$ & 0.908 & $0.671-1.228$ \\
\hline Saku & $97(14.4)$ & $576(85.6)$ & 1 & \\
\hline Nagaoka & $106(11.9)$ & $787(88.1)$ & 0.846 & $0.630-1.136$ \\
\hline
\end{tabular}

Abbreviations: SAR, seasonal allergic rhinitis; CI, confidence interval [ ]: adjusted data, ${ }^{\star}$ : data adjusted for sex and city

\section{Discussion}

The present study's results showed that oral symptoms were experienced by $15.6 \%$ of Japanese children aged 7-15 years. There was between-region variation in the prevalence of oral symptoms, even though the 4 cities are located relatively close to each other, indicating that the prevalence of oral symptoms is likely influenced by SAR and factors such as climate, vegetation, and living conditions.

The prevalence of SAR in the Japanese population was reported at $29.8 \%$ in $2008,{ }^{11}$ with an increase by $10 \%$ since 1998 . The prevalence of SAR among adult populations in 4 regions with distinct climate in Japan, namely, Tokyo, Osaka, Hokkaido, and Okinawa, were reported at $33.8 \%, 27.4 \%, 18.8 \%$, and $7.4 \%$, respectively. ${ }^{11} \mathrm{~A}$ study on the prevalence of allergic diseases among school children aged 7-12 years in a western district of Japan showed large differences in SAR prevalence; specifically, 10.0\%, 8.7\%, 19.7\%, and 1.0\% in Fukuoka, Nagasaki, Hyogo, and Okinawa, respectively. ${ }^{19}$ In addition to the differences in the prevalence of a specific diagnosis, regional differences in pollen sensitization rates among adults and children have been reported in Japan..$^{20}$ The present study revealed large variations in SAR prevalence in 4 cities of central Japan, which have different climate characteristics even though they are in geographical proximity to each other.

Although OAS prevalence is expected to increase, there have been few studies on OAS prevalence in children residing in regions with distinct climate conditions. For example, the territory of Italy covers a significant stretch of land from north to south; OAS prevalence among children with SAR aged 4-18 years have been reported at $30.4 \%, 22.2 \%$, and $16.9 \%$ in northern, central, and southern Italy, respectively. ${ }^{21}$ In the present study, there was a close correlation between SAR and the prevalence of oral symptoms, suggesting that SAR might affect the development of oral symptoms in these regions. 
As white birch, one of the best-known causes of OAS, occurs naturally only in Saku, we had expected that the prevalence of oral symptoms would be highest in this city. Contrary to our expectations, the prevalence of oral symptoms was the highest in Maebashi. Concurrently, most children with SAR included in the present study were affected by Japanese cedar pollinosis. Japanese cedar pollen has been shown to be molecularly associated with OAS triggered by tomatoes. ${ }^{10}$ In the present study, oral symptoms related to tomato ingestion were reported by only $7 \%$ of respondents, which was a lower proportion than the proportions of respondents reporting symptoms triggered by kiwi (29.7\%), pineapple (25.3\%), and melon (16.4\%). Similar tendencies have been previously reported in other surveys of the Japanese population. ${ }^{16,17}$ However, crossallergenicity has not been demonstrated between Japanese cedar pollen and these fruits. Therefore, the mechanism of comorbidity between Japanese cedar pollinosis and OAS remains unknown.

Previous studies have suggested accelerated expansion of sensitization to multiple allergens in patients already sensitized to one allergen. ${ }^{22}$ Sensitization to Japanese cedar pollen might prime patients to become sensitized to other pollen types, such as birch pollen. However, as birch pollen or pollen from closely related trees is not as abundant locally as is Japanese cedar pollen, symptoms caused by these types of pollen might not be sufficient to be recognized. Future research should compare rates of sensitization to multiple types of pollen between participants with and those without Japanese cedar pollinosis.

This study has several limitations. First, this study was a questionnaire-based study. Second, sensitization was not directly tested. In non-SAR-diagnosed children, 10.2\% reported OAS-symptoms. Currently, it is unclear whether those children are sensitized to food or pollens. They might be sensitized to neither food nor pollens, or they may be sensitized to both but have subclinical SAR-related symptoms. Future studies should involve sensitization testing such as IgE measurement or the skin prick test to validate these results. Third, the questionnaire intake season was September to February. This is not a typical season for pollinosis, which might have caused bias in participants' recalling symptoms of pollinosis. Therefore, it might affect the results in morbidity. Fourth, selection of schools was not randomized, which could cause bias in the study.

\section{Conclusions}

The present questionnaire-based study demonstrated that the prevalence of oral symptoms among children in central Japan was $15.8 \%$ and was closely correlated with SAR prevalence. This is the first report of regional differences in the prevalence of oral symptoms among Japanese children. These results can be a basis for further research on prevalence transition, pollen sensitization, and OAS. They can also support the development of prevention and therapeutic strategies for these diseases.

\section{Conflicts of interest}

The authors declare no conflict of interest in relation to this work.

\section{Author contributions}

TT designed the survey. MO and TT performed the survey and drafted the manuscript. MO, TT, YN, HY, KS, SY, and HA analyzed the data. All authors reviewed and edited the manuscript and approved the final version of the manuscript. All authors agree to be accountable for all aspects of this work and for any questions related to its accuracy or integrity.

\section{Acknowledgements}

We would like to thank Ms. Kiyoe Ishii for her excellent assistance with the preparation of this manuscript, and Editage (www.editage.com) for English language editing.

\section{References}

1. Amlot PL, Kemeny DM, Zachary C, Parkes P, Lessof MH. Oral allergy syndrome (OAS): symptoms of IgE-mediated hypersensitivity to foods. Clin Allergy. 1987; 17:33-42.

2. Price A, Ramachandran S, Smith GP, Stevenson ML, Pomeranz MK, Cohen DE. Oral allergy syndrome (pollen-food allergy syndrome). Dermatitis. 2015;26:78-88.

3. Kondo Y, Urisu A. Oral allergy syndrome. Allergol Int. 2009;58:485-91.

4. Ma S, Sicherer SH, Nowak-Wegrzyn A. A survey on the management of pollen-food allergy syndrome in allergy practices. J Allergy Clin Immunol. 2003;112:784-8.

5. Ebisawa M, Ito K, Fujisawa T. Japanese guidelines for food allergy 2017. Allergol Int. 2017;66(2):248-64.

6. Kashyap RR, Kashyap RS. Oral allergy syndrome: an update for stomatologists. J Allergy (Cairo). 2015;2015:543928.

7. Vanekkrebitz M, Hoffmannsommergruber K, Machado M, Susani M, Ebner C, Kraft D, et al. Cloning and sequencing of Mal d 1, the major allergen from apple (Malus domestica), and its immunological relationship to Bet v 1, the major birch pollen allergen. Biochem Biophys Res Commun. 1995;214:538-51.

8. Ebner C, Birkner T, Valenta R, Rumpold H, Breitenbach M, Scheiner O, et al. Common epitopes of birch pollen and apples-studies by western and northern blot. J Allergy Clin Immunol. 1991;88:588-94.

9. Pauli G, Bessot JC, Dietemann-Molard A, Braun PA, Thierry R. Celery sensitivity: clinical and immunological correlations with pollen allergy. Clin Allergy. 1985;15:273-9.

10. Kondo Y, Tokuda R, Urisu A, Matsuda T. Assessment of cross-reactivity between Japanese cedar (Cryptomeria japonica) pollen and tomato fruit extracts by RAST inhibition and immunoblot inhibition. Clin Exp Allergy. 2002;32:590-4.

11. Baba K, Nakae K. [National epidemiological survey of nasal allergy 2008 (compared with 1998) in otolaryngologists and their family members.] [Prog Med] 2008;28(8):2001-12. [Article in Japanese]

12. Zhang Y, Zhang L. Increasing prevalence of allergic rhinitis in China. Allergy Asthma Immunol Res. 2019;11:156-69.

13. Li F, Zhou Y, Li S, Jiang F, Jin X, Yan C, et al. Prevalence and risk factors of childhood allergic diseases in eight metropolitan cities in China: a multicenter study. BMC Public Health. 2011;11:437.

14. Dunlop J, Matsui E, Sharma HP. Allergic rhinitis: environmental determinants. Immunol Allergy Clin North Am. 2016;36:367-77.

15. Adachi A, Horikawa T. [Local differences of oral allergy syndrome with pollinosis--a comparison between Hanshinkan and Higashiharima in south Hyogo.] [Arerugi.] 2006;55:811-9. [Article in Japanese]

16. Adachi A, Horikawa T. [Epidemiological survey of oral allergy syndrome with pollinosis and food-dependent exercise-induced anaphylaxis among elementary, junior high, and senior high school children in Higashi -Harima District of Hyogo]. J Environ Dermatol Cutan Allergol. 2007; 1(2):102-8. [Article in Japanese] 


\section{APJAI}

17. Taisuke K, Kana Y, Yamao W, Yoshikazu K. [A surveillance of fruits allergy in school children in Gamagori city Aichi prefecture]. Jpn J Pediatr Allergy Clin Immunol. 2015;29(5):676-84. [Article in Japanese]

18. R Core Team. R: A Language and Environment for Statistical Computing. R Foundation for Statistical Computing. Vienna, Austria; 2015

19. Nishima S, Odajima H, Ota K, Oka N, Okazaki K, Kanatani M, et al. A study on the prevalence of allergic diseases in school children in western districts of Japan. Jpn J Pediatr Allergy Clin Immunol. 2013;27:149-69.

20. Minami T, Fukutomi Y, Inada R, Tsuda M, Sekiya K, Miyazaki M, et al. Regional differences in the prevalence of sensitization to environmental allergens: analysis on IgE antibody testing conducted at major clinical testing laboratories throughout Japan from 2002 to 2011. Allergol Int. 2019;68:440-9.
21. Mastrorilli C, Tripodi S, Caffarelli C, Perna S, Di Rienzo-Businco A, Sfika I, et al. Endotypes of pollen-food syndrome in children with seasonal allergic rhinoconjunctivitis: a molecular classification. Allergy. 2016;71:1181-91.

22. Matricardi PM, Bockelbrink A, Keil T, Gruber C, Niggemann B, Hamelmann E, et al. Dynamic evolution of serum immunoglobulin E to airborne allergens throughout childhood: results from the Multi-Centre Allergy Study birth cohort. Clin Exp Allergy. 2009;39:1551-7. 